\title{
A Single-Center Case Series of Endoscopically Treated Aorto-Gastrointestinal Fistula after Endovascular Aortic Repair: Surgery Is Still the Only Valid Solution
}

\author{
Alica Kubesch $^{\mathrm{a}}$ Oliver Waidmann ${ }^{\mathrm{a}}$ Irina Blumenstein ${ }^{\mathrm{a}}$ Wolf Otto Bechstein ${ }^{\mathrm{b}}$ \\ Mireen Friedrich-Rust ${ }^{a}$ Michael Jung ${ }^{a}$ Jörg Albert ${ }^{a, c}$ Johannes Hausmann ${ }^{\text {a, } d}$ \\ aDepartment of Internal Medicine 1, Goethe-University Hospital Frankfurt, Frankfurt, Germany; \\ ${ }^{b}$ Department of General and Visceral Surgery, Goethe-University Hospital Frankfurt, Frankfurt, Germany; \\ 'Department of Internal Medicine, Robert-Bosch-Hospital, Stuttgart, Germany; \\ ${ }^{\mathrm{d}}$ Department of Gastroenterology, St. Vinzenz-Hospital Hanau, Hanau, Germany
}

\section{Keywords}

Endoscopy · Endovascular aortic repair (EVAR) - TEVAR ·

Aortoesophageal fistula (AEF) - Stent · Fistula $\cdot$ OTSC $^{\circledR}$

\begin{abstract}
Introduction: Aortoesophageal fistula (AEF) is a life-threatening complication associated with endovascular aortic repair (EVAR) and occurs mostly in patients who undergo thoracic EVAR (TEVAR). To date, surgical treatment of AEF has been considered the most promising therapeutic approach. New endoscopic techniques could contribute to the therapy of AEF. The aim of this study was to analyze the outcome after endoscopic treatment of EVAR-associated AEF. Methods: All patients who received endoscopic diagnostics and/or therapy for AEF after EVAR/TEVAR in our center between 2010 and 2019 were evaluated. Results: Seven suitable patients were included. Six of them had undergone TEVAR and 1 had EVAR. Fistula occurred at an average of 307 days (range 21-2,774 days) post-EVAR. Endoscopic treatment was performed on 4 patients by using an over-the-scope clip $\left(\right.$ OTSC $\left.^{\circledR}\right)$. However, fistula recurred in all patients who were initially treated endoscopically. They were then treated either by sequential application of further OTSCs ${ }^{\circledR}$ or by implantation of a fully coated, self-expanding metal stent. One of these patients underwent a partial esophageal resection in a subsequent treatment approach. All patients died during the observational period, 5 as a direct consequence of
\end{abstract}

the AEF/aortoduodenal fistula and 2 due to comorbidities. The median survival time after fistula occurrence was 120 days (range 5-823 days). Conclusion: Endoscopic treatment of AEF with OTSC ${ }^{\circledR}$ should be considered as a possible interim solution, especially in patients with severe comorbidities. However, surgical remediation still appears to be the only procedure with intermediate to long-term therapeutic success.

C 2020 S. Karger AG, Basel

\section{Introduction}

Since its introduction in the early 1990s [1, 2] minimally invasive endovascular aortic repair (EVAR) therapy for thoracic and abdominal aortic diseases has been established as a reliable alternative to open surgery not only in high-risk patients [3]. A rare but life-threatening complication after EVAR is the development of an aortointestinal fistula, most commonly reported after thoracic EVAR (TEVAR) as an aortoesophageal fistula (AEF). AEF occurs in $0.5-1.7 \%[4,5]$ of cases and is fatal in $100 \%$ if untreated. Due to the increasing number of patients and longer follow-up intervals, there has been an increase in reports of AEF after TEVAR in recent years $[6,7]$. Risk factors for the occurrence of AEF are prosthesis infection, pseudoaneurysms, emergency surgery, and perioperative complications $[6,8]$. To date, surgical treatment of AEF 
remains the only promising therapeutic approach [9-11]. However, as such complications are still relatively rare, the reports often include only a few patients and focus mainly on surgical management rather than the evaluation of endoscopic therapy approaches. The development of alternative therapy approaches to surgery is of clinical relevance since many patients are not candidates for surgical treatment as they suffer from severe comorbidities and are very often in a severely reduced general condition [8].

The use of so-called over-the-scope clips $\left(\right.$ OTSC $^{\circledR}$, Ovesco Endoscopy AG, Germany) placed endoscopically as a treatment modality for AEF has been poorly described. The aim of our study was to investigate whether endoscopic treatment, especially the use of OTSCs, presents a viable alternative for patients with comorbidities that preclude surgical repair of the defect.

\section{Materials and Methods}

Study Population and Definition of Aortointestinal Fistula

All patients who received endoscopic diagnostics and/or therapy for AEF after TEVAR and aortoduodenal fistula post-EVAR in our center between 2010 and 2019 were included in this retrospective study. All relevant information about the patients was obtained with the help of the digital medical records. AEF/aortoduodenal fistula was defined as a communication between the esophagus and the thoracic aorta after TEVAR or communication between the duodenum and aorta post-EVAR. All patients in whom AEF was already present at the time of the initial TEVAR or EVAR implantation were excluded from our analysis.

\section{OTSC and Stent Implantation}

OTSCs were used to close AEF according to the technique recommended by the manufacturer. In contrast to the regular clips used for hemostasis, OTSCs are claw-like and thus guarantee better closure and stability in the area of the defect. However, it is crucial that there is viable tissue surrounding the defect to ensure secure OTSC placement and closure. The fully covered self-expandable metallic stents (cSEMS) that are used have a double-Slike structure (Niti-S esophageal stent, Taewoong Medical Seoul, South Korea), with the aim of preventing possible dislocation of the stent.

\section{Statistical Analysis}

Nominal data are presented as $n(\%)$, ordinal data as median (interquartile range [IQR]), and quantitative data as mean and SD, or if skewed as median (IQR). Statistical analyses were conducted using SPSS Statistics v22.0 (IBM Corp., Endicott, NY, USA).

\section{Results}

Characteristics of Patients and the Initial Intervention Seven male patients were identified and included in the analysis. Six suffered from AEF post-TEVAR and 1 patient from aortoduodenal fistula after EVAR. The median age was 71 years (range $56-78$ years). Four patients
Table 1. Characteristics of the 7 patients

\begin{tabular}{ll}
\hline Demographics & \\
Median age, years (IQR) & $71(56-78)$ \\
Male sex, $n$ & 7 \\
\hline & $n$ (patient Nos.) \\
\hline Chronic health conditions and risk factors & \\
Hypertension & $4(\mathrm{P} 1-\mathrm{P} 3, \mathrm{P} 6)$ \\
Coronary artery disease & $3(\mathrm{P} 1, \mathrm{P} 6, \mathrm{P} 7)$ \\
Previous cardiac surgery/intervention & $2(\mathrm{P} 6, \mathrm{P} 7)$ \\
Previous aortic surgery/intervention & $1(\mathrm{P} 1)$ \\
Other & $1(\mathrm{P} 4)$ \\
Underlying pathology & $5(\mathrm{P} 1-\mathrm{P} 4, \mathrm{P} 7)$ \\
Aneurysm & $2(\mathrm{P} 5, \mathrm{P} 6)$ \\
Acute type B aortic dissection & $2(\mathrm{P} 5, \mathrm{P} 6)$ \\
Initial emergency surgery & \\
\hline
\end{tabular}

Table 2. Aortointestinal characteristics fistula in the 7 patients

\begin{tabular}{ll}
$\begin{array}{l}\text { Median number of days after initial TEVAR } \\
\text { surgery (IQR) }\end{array}$ & $307(21-2,774)$ \\
\hline & $n$ (patient Nos.) \\
\hline $\begin{array}{l}\text { Clinical presentation, } n \\
\text { Infection }\end{array}$ & $3(\mathrm{P} 2, \mathrm{P} 3, \mathrm{P} 7)$ \\
Hematemesis/hemoptysis & $2(\mathrm{P} 2, \mathrm{P} 3)$ \\
Shock & $1(\mathrm{P} 5)$ \\
Dyspnea & $4(\mathrm{P} 1-\mathrm{P} 4)$ \\
Other & $3(\mathrm{P} 1, \mathrm{P} 6, \mathrm{P} 7)$ \\
Diagnostics, $n$ & $7(\mathrm{P} 1-\mathrm{P} 7)$ \\
Computed tomography & $7(\mathrm{P} 1-\mathrm{P} 7)$ \\
Endoscopy & $7(\mathrm{P} 1-\mathrm{P} 7)$ \\
C-reactive protein, mg/dL &
\end{tabular}

suffered from underlying chronic conditions and possible risk factors for aneurysm or dissection, specifically, arterial hypertension, coronary artery disease, previous cardiac/aortic surgery/intervention, and untreated syphilis. The underlying pathology causing the initial need for endovascular repair was an aneurysm in 5 patients and an acute type $\mathrm{B}$ dissection of the descending thoracic aorta in 2 . In 2 patients, the initial intervention was performed in an emergency. One patient had already undergone previous vascular surgery, namely infra-aortic vascular repair (Table 1).

\section{Symptoms and Diagnosis of Aortointestinal Fistula}

Aortoduodenal fistula was diagnosed on average 307 days after initial EVAR implantation (range 21-2,774 days). Symptoms of the fistula included signs of infection, reduced general condition, hematemesis or hemoptysis, hemorrhage, and dyspnea. All patients received a full laboratory workup including markers of systemic inflammation, computed tomography (CT), and peroral esophagogastroduodenoscopy (EGD) to confirm the fis- 
Fig. 1. An overview of treatment approaches and survival for each patient. d, Days.

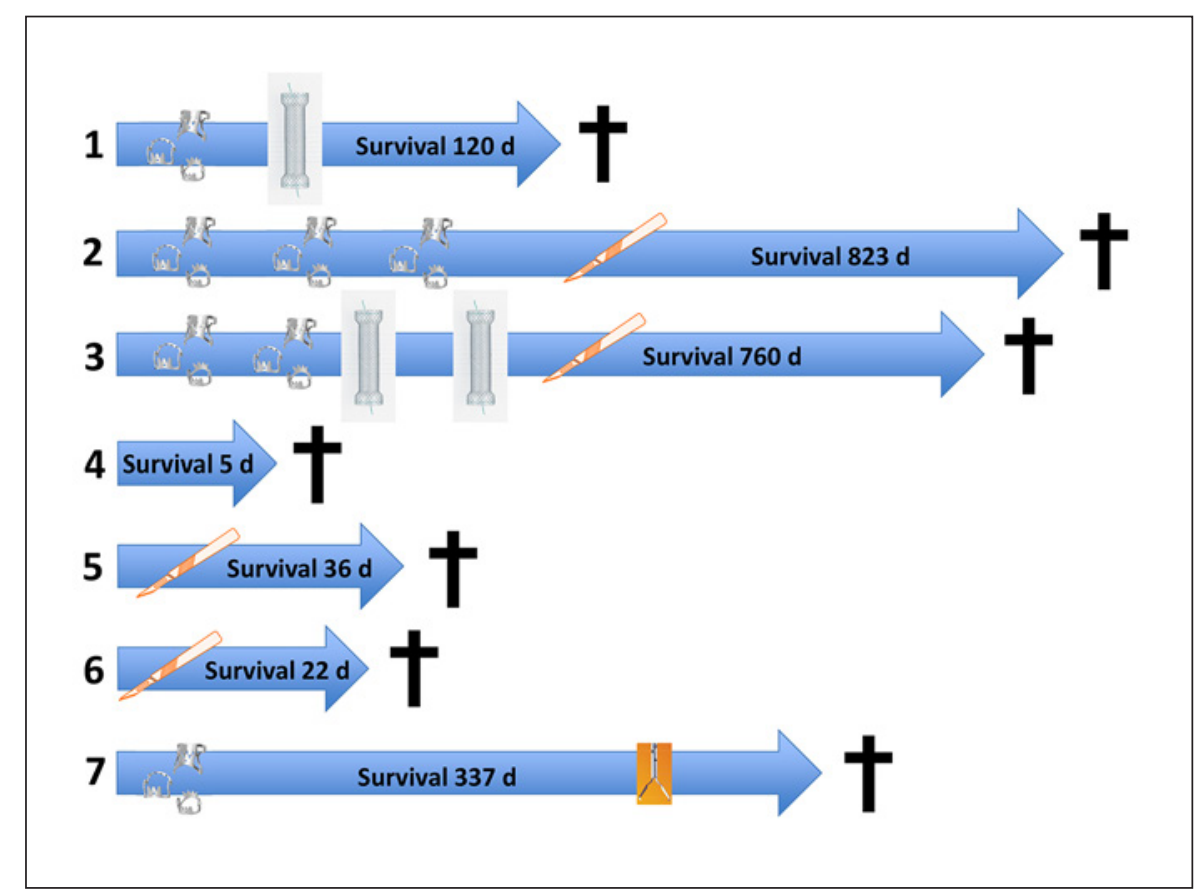

tula. One patient initially presented with severe mediastinitis as well as spinal cord abscess (Table 2).

\section{General Management, Therapy, and Outcome}

In 4 patients (Patients 1, 2, 3, and 7), an endoscopic attempt was decided at an interdisciplinary review, as the patients were all in a reduced general condition and primarily not fit for extensive reconstructive surgery. The other 3 patients were not eligible for endoscopic therapy, as the defect was either too big or fistula closure was not technically feasible or advised. Thus, patients 4, 5 and 6 either underwent primary surgical repair or received palliative treatment. The median survival time after fistula occurrence was 120 days (range 5-823 days). A detailed report is provided in the next paragraph. For an overview of the treatments and survival, please see Figure 1 and Table 3.

\section{Detailed Treatment Report}

All patients were in a reduced state and underwent diagnostic endoscopy to verify the suspected AEF to evaluate if an endoscopic treatment regimen appeared feasible. Four patients were initially treated endoscopically, but in all these cases, a recurrence of the AEF was reported. Detailed information on the course of treatment is provided in the following section.

\section{Patient 1}

This patient underwent an elective TEVAR procedure due to an aortic aneurysm. He presented 307 days later with dyspnea and thoracic pain. A CT scan showed a leak of the aortic stent and endoscopy showed a stenosis of the
Table 3. Management and outcome $(n=7)$

\begin{tabular}{lc}
$\begin{array}{l}\text { Management } \\
\text { Conservative }\end{array}$ & $1(\mathrm{P} 4)$ \\
Isolated clip application & $1(\mathrm{P} 7)$ \\
Esophageal stenting and clip application & $1(\mathrm{P} 1)$ \\
$\begin{array}{l}\text { Esophageal stenting, clip application, and } \\
\quad \text { esophagectomy }\end{array}$ & $1(\mathrm{P} 3)$ \\
Clip application and surgery & $1(\mathrm{P} 2)$ \\
Isolated esophagectomy & $1(\mathrm{P} 5)$ \\
Other surgery $^{\mathrm{a}}$ & $1(\mathrm{P} 6)$ \\
\hline Outcome $^{\text {Median survival, days (IQR) }}$ & \\
Deaths due to the fistula & $120(5-823)$ \\
Deaths due to comorbidities &
\end{tabular}

Values express $n$ (specific patients affected), unless otherwise indicated.

${ }^{a}$ Vascular emergency surgery; ${ }^{b}$ metastasized prostate cancer and hyperosmolar coma.

esophagus due to a hematoma but no AEF. Consequently, an emergency restent implantation was performed. Four weeks after the intervention, a fistula at $25 \mathrm{~cm}$ ab ore was confirmed during endoscopy and closed with an OTSC $^{\circledR}$. Another 4 weeks later, a videofluoroscopic swallow study showed persisting leakage. Another endoscopy was performed and an esophageal perforation close to the OTSC was observed. The patient received a cSEMS (100 $\mathrm{mm}$ in length and $24 \mathrm{~mm}$ in diameter), and complete coverage of the defect was documented. To ensure enteral nutrition, he received a gastrostomy tube placed after gastropexy. After 6 weeks, he presented with pneumonia. 
Fig. 2. Images from an esophagram performed after repeated endoscopic treatment of AEF with OTSC (Patient 2). a Leakage of contrast agent close to previously applied OTSC. b Sufficient defect closure was achieved with further OTSC.
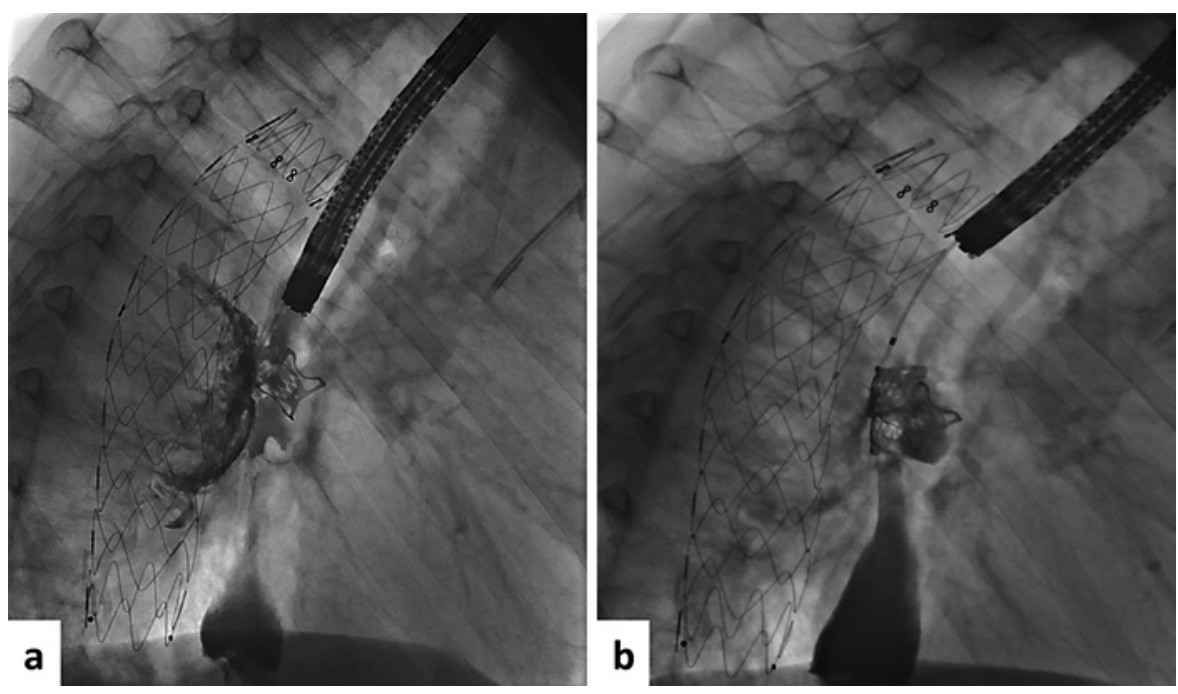

Fig. 3. A series of images of endoscopic treatment (Patient 3). a AEF before treatment. b Initial AEF closure using OTSC. c Recurrence of AEF in proximity to OTSC. d Treatment of AEF recurrence with cSEMS.

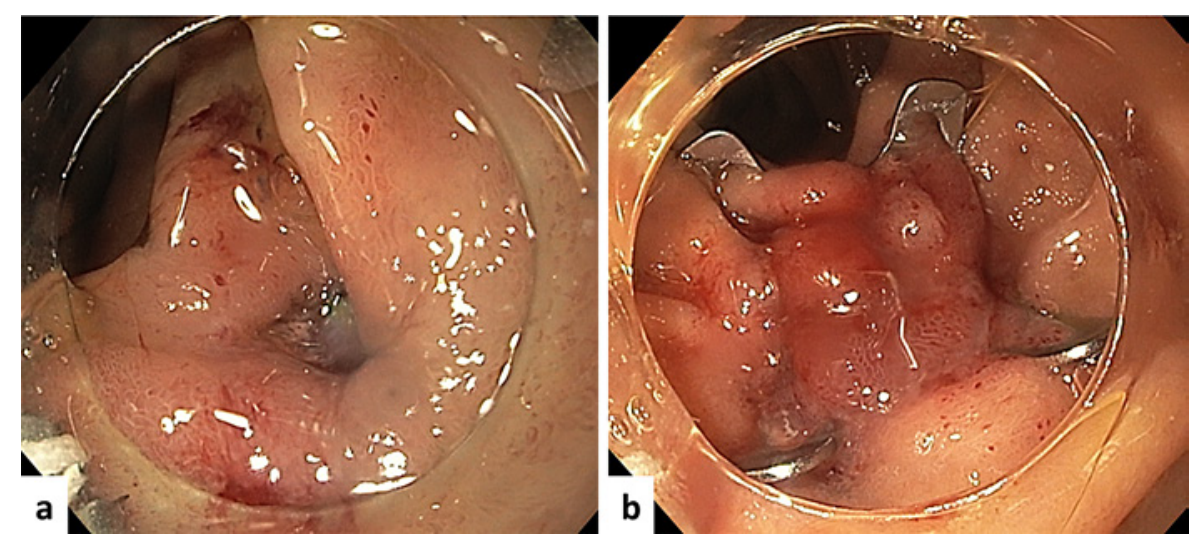

Bronchoscopy confirmed compression of the left main bronchus by the aortic stent. After careful consideration and interdisciplinary discussion, a conservative course of treatment was agreed upon. The patient then died of sepsis due to recurring pneumonia.

\section{Patient 2}

This patient initially received TEVAR for type B aortic dissection. He became symptomatic 2,774 days after the initial procedure. He presented with anemia of unknown origin, dyspnea, and hemoptysis. CT scan revealed an infection close to the prosthesis as well as the formation of a spine abscess (BWK5-7). EGD was performed, confirming a fistula in the upper third of the esophagus. Primary closure of the fistula was performed with an OTSC, and fluoroscopy confirmed complete closure of the defect. CT-guided abscess drainage was performed, and the patient was discharged with antibiotic treatment. Two subsequent swallow studies did not show any signs of fistulation. Almost 1 year later, the patient presented with signs of infection and mediastinitis, confirmed by CT scan. Endoscopy showed a recurrent esophageal fistula, that was again closed with an OTSC (Fig. 2). Several control endoscopies showed no reoccurrence of the fistula. Ten months later, asymptomatic reoccurrence of the AEF was detected via routine control CT, and defect closure was again achieved with an OTSC. Two months later, the patient suffered from upper gastrointestinal (GI) bleeding close to the OTSC, which was temporarily controlled with a fibrin glue injection. However, he ultimately underwent a discontinuity resection of the esophagus, and a cervical anastomosis was planned for a later time point due to persistent bleeding. He died 1 year later from prostate cancer, i.e., not from initial vascular condition, and associated complications.

\section{Patient 3}

This patient also underwent TEVAR for an aortic aneurysm. Seventy-seven days after implantation, he presented with hemoptysis and a reduced general condition. CT scan detected no leakage. EGD found an AEF, $8 \mathrm{~mm}$ in size, that was then closed with an OTSC. Nineteen weeks later, another OTSC was placed for a persisting fistula. Sixteen weeks later, a cSEMS was placed endoscopi- 
Fig. 4. Aortoduodenal fistula and respective endoscopic treatment (Patient 7). a Descending duodenum with fistula. b-d Aortoduodenal fistula after closure with OTSC.
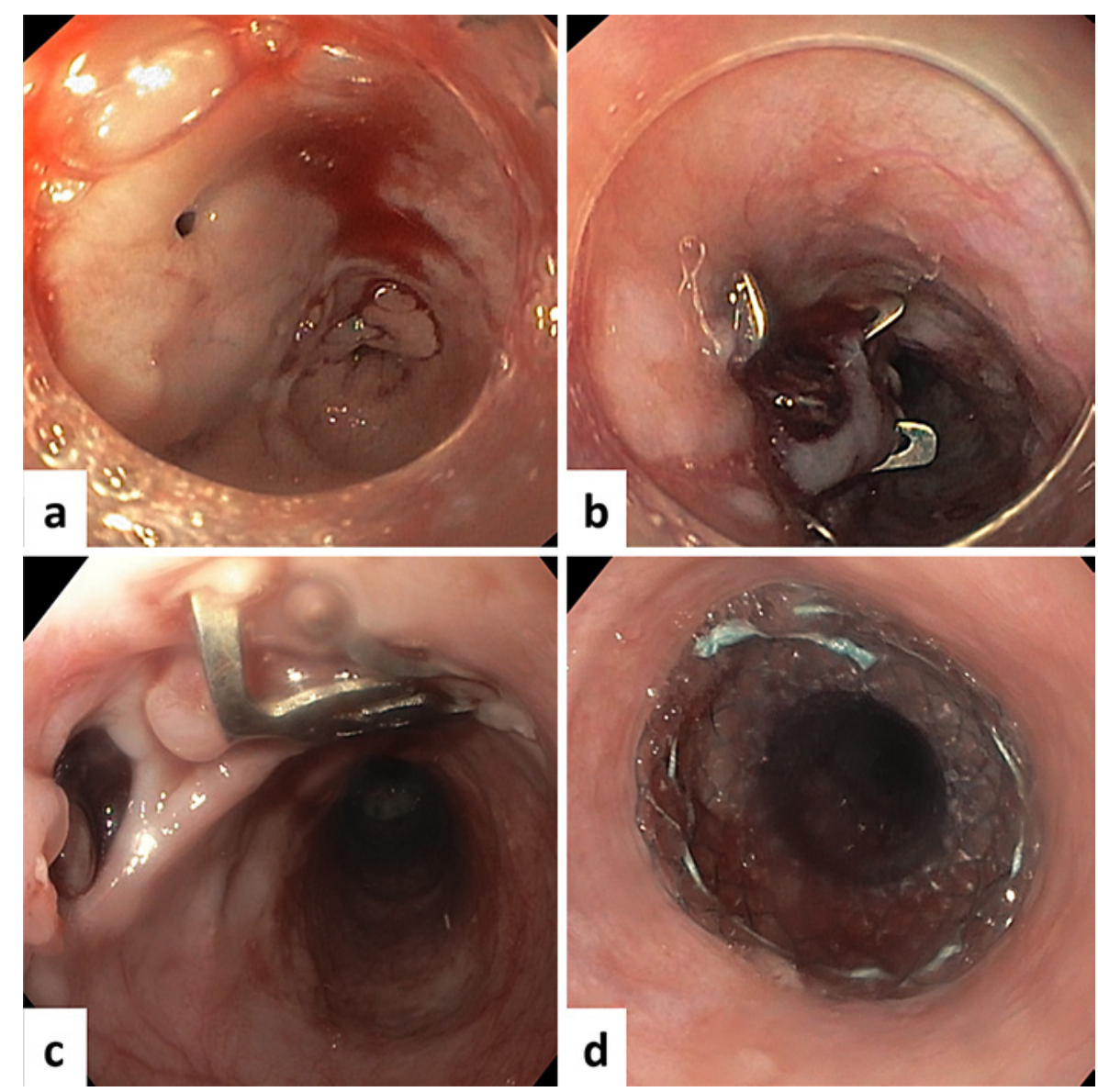

cally, covering the persisting defect. Five days afterwards, the cSEMS was removed and the defect was closed with 2 OTSCs to prevent pressure necrosis of the esophageal wall.

The patient underwent a thoracotomy and resection of segment 6 of the left lung for recurring pulmonary bleeding, and another endoscopic esophageal stent placement. Three endoscopies were performed for stent repositioning, including with metal clip fixation (Fig. 3). Three months later, the stent was removed at the patient's wish. He then underwent discontinuity resection of the esophagus. As he was in a poor overall state with a reduced lung capacity, the surgery was supported by extracorporeal membrane oxygenation (ECMO). After a prolonged stay in the ICU, he was discharged into a rehabilitation facility. He was readmitted to the hospital 10 months after the esophagectomy with hyperosmolar coma as a first manifestation of diabetes mellitus, with a fatal outcome.

\section{Patient 4}

This patient presented with dyspnea and a reduced overall state. He had initially undergone EVAR 3 years prior and had already been treated for an endoleak 1 year after this surgery. Interestingly, he also had untreated syphilis, possibly the cause of his aneurysm. An abdomi- nal CT scan showed signs of stent-graft infection (i.e., air entrapment) and spondylodiscitis. Due to his reduced overall state, he was not eligible for surgical repair and was given antibiotics. He remained in the ICU and later showed signs of an upper GI bleed. EGD revealed a large esophageal defect with no minimally invasive treatment option. He died 5 days later from septic shock.

\section{Patient 5}

This patient was admitted with a covered ruptured aortic aneurysm. Emergency operative aortic repair with a prosthesis was performed. Subsequently, he suffered from acute respiratory distress syndrome (ARDS) and was dependent on an extracorporeal life support system (ECLS) from which he could be successfully weaned. He then suffered from repeated upper GI bleeding events. EGD showed an esophageal fistula $25-28 \mathrm{~cm}$ ab ore. Due to the size of the defect, an endoscopic closure was not feasible and a nasogastric tube was inserted. Follow-up EGD showed the persisting fistula. Application of contrast agent also revealed a communication with the left main bronchus. Interestingly, a previously conducted bronchoscopy had not revealed fistulation. After an interdisciplinary discussion of the case, the patient underwent esophagectomy, and the pulmonary defect was closed 
with the help of a latissimus flap. Before surgery, the patient was connected to ECMO from which he could not be successfully weaned this time. Ultimately, the patient went into multiorgan failure and died 36 days after the fistula occurrence.

\section{Patient 6}

This patient complained of abdominal pain when he first presented at a peripheral hospital. He had already undergone a 4-way endoprosthetic repair of a thoracoabdominal aortic aneurysm 2 years previously. An abdominal CT revealed a hematoma close to the prosthesis, with luxation of the renal branch and a type III endoleak. He underwent immediate surgery to repair the covered aortic perforation, with partial resection of the prosthesis, aortomesenterial and renal bypass, and endovascular repair of the aneurysm. After initial recuperation, an intraabdominal infection was suspected on the CT scan (i.e., via contrast enhancement in the small bowel). He underwent an explorative laparotomy that ruled out intra-abdominal pathology. He remained in the ICU, and upper GI bleeding was suspected. EGD initially showed ischemia of the posterior gastric wall as well as a gastroesophageal fistula at $35 \mathrm{~cm}$. Palliative therapy was decided upon after an interdisciplinary discussion.

\section{Patient 7}

This patient initially underwent EVAR for an infrarenal aortic aneurysm. He presented 3 years later with a type 1a endoleak which, again, was electively treated. Two years after the second intervention, abscess formation was suspected on a follow-up CT in the former aneurysm sac. Endoscopic examination detected a bleeding aortoduodenal fistula with suspected communication to the abscess. Successful closure of the defect was performed with an OTSC (Fig. 4). The patient was discharged with a long-term course of oral antibiotics. The 6-week control showed no significant improvement, and removal of the infected aortal prosthesis was recommended. However, the patient declined surgical treatment and was discharged from hospital. Sixteen weeks later, he was admitted to another hospital with GI bleeding. EGD revealed recurrence of the aortoduodenal fistula. Defect closure and hemostasis were performed endoscopically with 5 conventional clips (i.e., not OTSC). He died 2 months later due to complications associated with the fistula.

\section{Discussion}

EVAR has revolutionized the treatment of patients with aortic aneurysms and aortic dissections. Especially for unfit patients, it has become a valid alternative for the treatment of aortic pathologies instead of open surgical repair $[1,10]$. With the rise in the number of procedures, the reports on complications have increased over the last decade $[6,11-13]$. Managing the complications associated with advanced procedures in increasingly frail patients, in particular, is one of the great challenges of modern medicine.

In patients treated with EVAR, a rare but often lethal complication is the formation of an AEF. In patients receiving TEVAR, an AEF incidence of $1.9 \%$ was reported in a cohort of 268 patients [6]. The treatment of choice, which guarantees the best outcome, is open operative reconstruction. However, many patients are not eligible for this due to cardiovascular and other comorbidities [14, 15]. Alternative options, either as bridging to surgery or a palliative measure, are needed. One endoscopic approach is the use of an esophageal cSEMS to cover the area of the defect, and several publications have reported at least short-term success $[8,16,17]$. However, the possible formation of pressure-induced necrosis and frequent stent dislocation limit its application [18]. Closing defects endoscopically with metal clips has become more realistic and has proved consistently successful with the introduction of OTSC in 2006 [19].

In contrast to regular through-the-scope clips, OTSCs have a larger span/diameter and their claw-like structure makes it easier to grasp the surrounding tissue. Several studies have reported promising results $[19,20]$, and OTSCs are now widely used in clinical practice for different indications [21]. As AEF post-TEVAR is a rare complication and the data on symptoms, management, and outcome are still scarce, we wanted to share our experience with a particular focus on endoscopic management. To the best of our knowledge, we are first to report on the use of the OTSC to manage AEF [22].

Seven patients suffering from postinterventional AEF or aortoduodenal fistula were identified and included in our study. The reported underlying comorbidities (i.e., hypertension, coronary artery disease, and previous cardiac/vascular intervention) and pathologies (i.e., aneurysm and acute type $\mathrm{B}$ dissection) were in line with the literature [9]. One patient suffered from untreated syphilis which caused aneurysm formation in the thoracic aorta. In 5 cases, the initial intervention was elective; in 2 cases, it was performed as an emergency intervention, described as another risk factor for later complications [8]. In our collective, the median time to diagnosis of AEF was 307 days (range 21-2,774 days); other studies reported this to be between 1 week and $>4$ years $[9,11,23,24]$. Of note, 1 patient presented with AEF 2,774 days after TEVAR, demonstrating that AEF may occur even years after implantation and should be kept in mind when patients present with symptoms suspicious for AEF. The Chiari triad most prominently describes the symptoms of AEF as mid-thoracic pain and sentinel hematemesis, followed 
by exsanguinating hemorrhage after a variable asymptomatic period [25]. However, this can prove to be challenging when the patient has comorbidities that could also explain the reported symptoms. In 1 of our patients, exacerbation of COPD was diagnosed when he presented with dyspnea, signs of infection, and a reduced overall state. Thus, although it is a rare complication, AEF should be on the physician's radar in post-EVAR patients. The primary symptoms in our patients were signs of infection. In line with the literature, prosthetic infection is one of the major risk factors for AEF, with an incidence of 0.55\% [6]. In their review article, Canaud et al. [26] showed that, in $>40 \%$ of the patients with post-TEVAR AEF, micro-organisms were detected, and in patients with virulent inflammation, pathogens could be isolated. Another study also detected a virulent pathogen, Coxiella burnetti, in aneurysmal graft infection [27].

It must be noted that all our patients presented with subacute symptoms of AEF (i.e., not analogous to the Chiari triad) and had relevant comorbidities. Thus, after interdisciplinary discussion, the primary goal was to treat the patients as minimally invasively as possible, as they were considered unfit for surgery. Four patients were found to be eligible for endoscopic fistula treatment after the index EGD. Endoscopic treatment was primarily intended to be a bridging method to surgery at a later time. In all cases, closure of the defect was initially conducted with OTSC, and no immediate adverse events were reported. The rationale behind this approach was that, in our experience, as well as going by the literature, the implantation of an esophageal stent might lead to dislocation and increase the risk of tissue damage due to stenton-stent friction.

Furthermore, stent ingrowth has been reported, even when using a cSEMS, due to hypergranulation or defect in-stent coverage. Two of our patients still received esophageal stenting, as AEF persisted after OTSC application. After initial endoscopic treatment and stabilization, 2 patients (Patients 2 and 3 ) underwent surgical reconstruction for definitive treatment. Both of them died of causes unrelated to AEF complications or consecutive treatment (Patient 2: prostate cancer; Patient 3: diabetic hyperosmolar coma). Thus, in 2 patients, OTSC application was a useful short-term bridging measure to surgery.

Our study has underlined that the application of OTSC to close defects has diverse challenges. Tissue fibrosis can impede grasping the lesion (i.e., fistula) and thus render adequate OTSC application more difficult. Large defects can also limit the use of the OTSC [28]. A recent review on OTSC application in 1,517 cases considered fistula closure as 1 of the less promising indications [21].

The retrospective nature and small sample size are surely limitations of our study. Furthermore, our patient collective was heterogeneous in terms of the presentation, symptoms, manifestation of the fistula and, lastly, the treatment. This can, in part, be explained by the rarity of this complication.

Nevertheless, we believe our study does provide further insight into the management and treatment of a rare complication that can occur after EVAR. Although endoscopic treatment does not appear to be an option for definitive treatment, especially if the prosthesis is infected, it could be considered as an interim solution in patients unable to undergo immediate surgical repair. Though our patients did have fistula recurrence after their endoscopic treatment, preliminary defect closure was feasible and lasted for up to 348 days. We therefore believe the endoscopic approach, especially the use of the OTSC, is an opportunity to gain time and improve the patient's overall state in the short term, thereby granting the possibility for them to recuperate before undergoing an extensive surgical repair. The treatment of AEF post-TEVAR is usually a highly individual matter and should be discussed on an interdisciplinary level as well as with the patient, in order to determine the best course of action.

\section{Statement of Ethics}

Approval for this study was obtained from the local institutional review board (ethics committee) of the University Hospital Frankfurt (file No. 178/19). As this was a retrospective study, written informed consent was not needed.

\section{Disclosure Statement}

The authors declare that they have no conflicts of interest.

\section{Funding Sources}

The authors received no funding for this study.

\section{Author Contributions}

The authors contributed to the manuscript by planning the study (O.W., J.H., and J.A.), collecting the data (J.H., A.K., O.W., I.B., J.A., W.O.B., and T.S.R.), analysis and interpretation of data (J.H., A.K., M.J., J.A., and O.W.), and preparation (J.H. and A.K.), and revision of the manuscript (all authors).

\section{References}

\footnotetext{
1 Dake MD, Miller DC, Semba CP, Mitchell RS, Walker PJ, Liddell RP. Transluminal placement of endovascular stent-grafts for the treatment of descending thoracic aortic aneurysms. N Engl J Med. 1994 Dec;331(26):172934.

2 Parodi JC, Palmaz JC, Barone HD. Transfemoral intraluminal graft implantation for abdominal aortic aneurysms. Ann Vasc Surg. 1991 Nov;5(6):491-9.
} 
3 Salata K, Hussain MA, de Mestral C, Greco E, Aljabri BA, Mamdani M, et al. Comparison of Outcomes in Elective Endovascular Aortic Repair vs Open Surgical Repair of Abdominal Aortic Aneurysms. JAMA Netw Open. 2019 Jul;2(7):e196578.

4 Lawrie GM, Earle N, De Bakey ME. Evolution of surgical techniques for aneurysms of the descending thoracic aorta: twenty-nine years' experience with 659 patients. J Card Surg. 1994 Nov;9(6):648-61.

5 Svensson LG, Crawford ES, Hess KR, Coselli JS, Safi HJ. Experience with 1509 patients undergoing thoracoabdominal aortic operations. J Vasc Surg. 1993 Feb;17(2):357-68.

6 Eggebrecht H, Mehta RH, Dechene A, Tsagakis K, Kühl H, Huptas S, et al. Aortoesophageal fistula after thoracic aortic stent-graft placement: a rare but catastrophic complication of a novel emerging technique. JACC Cardiovasc Interv. 2009 Jun;2(6):570-6.

7 Porcu P, Chavanon O, Sessa C, Thony F, Aubert A, Blin D. Esophageal fistula after endovascular treatment in a type B aortic dissection of the descending thoracic aorta. J Vasc Surg. 2005 Apr;41(4):708-11.

8 Tao M, Shlomovitz E, Darling G, Roche-Nagle G. Secondary aorto-esophageal fistula after thoracic aortic aneurysm endovascular repair treated by covered esophageal stenting. World J Clin Cases. 2016 Aug;4(8):233-7.

9 Czerny M, Eggebrecht H, Sodeck G, Weigang E, Livi U, Verzini F, et al. New insights regarding the incidence, presentation and treatment options of aorto-oesophageal fistulation after thoracic endovascular aortic repair: the European Registry of Endovascular Aortic Repair Complications. Eur J Cardiothorac Surg. 2014 Mar;45(3):452-7.

10 Walsh SR, Tang TY, Sadat U, Naik J, Gaunt $\mathrm{ME}$, Boyle JR, et al. Endovascular stenting versus open surgery for thoracic aortic disease: systematic review and meta-analysis of perioperative results. J Vasc Surg. 2008 May; 47(5):1094-8

11 Chiesa R, Melissano G, Marone EM, Marrocco-Trischitta MM, Kahlberg A. Aorto-oesophageal and aortobronchial fistulae following thoracic endovascular aortic repair: a national survey. Eur J Vasc Endovasc Surg. 2010 Mar;39(3):273-9.
12 Lee SH, Song PS, Kim WS, Park KB, Choi SH. A case of stent graft infection coupled with aorto-esophageal fistula following thoracic endovascular aortic repair in a complex patient. Korean Circ J. 2012 May;42(5):366-8.

13 Eggebrecht H, Baumgart D, Radecke K, von Birgelen C, Treichel U, Herold U, et al. Aortoesophageal fistula secondary to stent-graft repair of the thoracic aorta. J Endovasc Ther. 2004 Apr;11(2):161-7.

14 Czerny M, Zimpfer D, Fleck T, Gottardi R, Cejna M, Schoder M, et al. Successful treatment of an aortoesophageal fistula after emergency endovascular thoracic aortic stent-graft placement. Ann Thorac Surg. 2005 Sep;80(3): 1117-20.

15 Kotelis D, Gombert A, Jacobs MJ. Treatment of post-thoracic endovascular aortic repair aorto-esophageal fistula-only radical surgery can be effective: techniques and sequence of treatment. J Thorac Dis. 2018 Jun; 10(6): 3869-73.

16 Onodera M, Inoue Y, Fujino Y, Kikuchi S, Endo S. A case of secondary aortoesophageal fistula inserted a covered self-expanding esophageal stent to control gastrointestinal bleeding. Case Rep Gastrointest Med. 2013; 2013:857135.

17 Stanger E, Johnson AM, Juneja Mucci J. Novel Management of an Aortoesophageal Fistula Prior to Definitive Surgical Repair. Clin Med Insights Case Rep. 2019 Apr; 12: 1179547619839710.

18 Mudumbi S, Velazquez-Aviña J, Neumann H, Kyanam Kabir Baig KR, Mönkemüller K. Anchoring of self-expanding metal stents using the over-the-scope clip, and a technique for subsequent removal. Endoscopy. 2014 Dec; 46(12):1106-9.

19 Kirschniak A, Kratt T, Stüker D, Braun A, Schurr MO, Königsrainer A. A new endoscopic over-the-scope clip system for treatment of lesions and bleeding in the GI tract: first clinical experiences. Gastrointest Endosc. 2007 Jul;66(1):162-7.

20 Wedi E, Gonzalez S, Menke D, Kruse E, Matthes $\mathrm{K}$, Hochberger J. One hundred and one over-the-scope-clip applications for severe gastrointestinal bleeding, leaks and fistulas. World J Gastroenterol. 2016 Feb;22(5):184453.
21 Kobara H, Mori H, Nishiyama N, Fujihara S, Okano K, Suzuki Y, et al. Over-the-scope clip system: A review of 1517 cases over 9 years. J Gastroenterol Hepatol. 2019 Jan;34(1):22-30.

22 Uno K, Koike T, Takahashi S, Komazawa D, Shimosegawa T. Management of aorto-esophageal fistula secondary after thoracic endovascular aortic repair: a review of literature. Clin J Gastroenterol. 2017 Oct;10(5):393-402.

23 Luehr M, Etz CD, Nozdrzykowski M, Garbade J, Lehmkuhl L, Schmidt A, et al. Emergency open surgery for aorto-oesophageal and aorto-bronchial fistulae after thoracic endovascular aortic repair: a single-centre experience†. Eur J Cardiothorac Surg. 2015 Feb; 47(2):374-82.

24 Cheng L, Zhu J, Liu X, Liu W, Hu H, Zhang J, et al. A Successful Three-Stage Surgical Treatment for Aortoesophageal Fistula After Thoracic Endovascular Aortic Repair and Esophageal Stent Repair. Ann Thorac Surg. 2016 Dec;102(6):e503-5.

25 Chiari H. Ueber Fremdkoerpeverletzung des Oesophagus mit Aortenperforation. Ber Klin Wochenschr. 1914;51:7-9.

26 Canaud L, Ozdemir BA, Patterson BO, Holt PJE, Loftus IM, Thompson MM. Retrograde aortic dissection after thoracic endovascular aortic repair. Ann Surg. 2014 Aug;260(2): 389-95.

27 Okwara CJ, Petrasek J, Gibson M, Burstein E. Secondary Aortoesophageal Fistula Associated with Aneurysmal Graft Infection by Coxiella burnetii. ACG Case Rep J. 2016 Apr;3(3): 169-71.

28 Kobara H, Mori H, Fujihara S, Nishiyama N, Chiyo T, Yamada T, et al. Outcomes of gastrointestinal defect closure with an over-the-scope clip system in a multicenter experience: an analysis of a successful suction method. World J Gastroenterol. 2017 Mar;23(9):1645-56.

29 Haito-Chavez Y, Law JK, Kratt T, Arezzo A, Verra M, Morino M, et al. International multicenter experience with an over-the-scope clipping device for endoscopic management of GI defects (with video). Gastrointest Endosc. 2014 Oct;80(4):610-22.

30 Law R, Wong Kee Song LM, Irani S, Baron TH. Immediate technical and delayed clinical outcome of fistula closure using an over-thescope clip device. Surg Endosc. 2015 Jul;29(7): 1781-6. 